\title{
Anthelmintic activity of plant aqueous extracts against Panagrellus redivivus in vitro
}

\author{
Atividade anti-helmíntica de extratos aquosos \\ de plantas contra Panagrellus redivivis in vitro
}

\author{
Cleonice Lubian'* (D), Danielle Dutra Martinha² (D), Roberto Portz² (D, Manoel Penachio Gonçalves² (D), \\ Sabrina Holz ${ }^{3}$ (D), Wesler Luiz Marcelino ${ }^{3}$ (D), Ana Claudia Constantino Nogueira 4 (D), \\ Renata Mori Thomé ${ }^{5}$ (D), Vivian Carré Missio ${ }^{2}$ (D), Juliano Cordeiro ${ }^{2}$ (D), Lorraine Tomim Feroldi ${ }^{2}$
}

\begin{abstract}
Control of phytonematodes is very hard and requires a combination of techniques to succeed. Alternative control through plant extracts may result in the discovery of nematicide substances. Research aimed at evaluating the effect of 33 plants submitted to aqueous extraction against Panagrellus redivivus in vitro. Concentrations were prepared at 1.25, 2.5, 5, 10, and 20\%. Monitoring happened at 0, 6, 12, 24 and 30 hours after preparation. Counting considered dead nematodes subtracted from alive ones. Juveniles were also counted, and extract efficiency was expressed in percentage of control or stimuli. Data were submitted to variance analysis. Significant results got with the Scott-Knott test (5\%), and multiple linear regression analysis. Extracts were observed acting as controllers, but also as stimulators to nematode reproduction. The best controlling performance was set by Carica papaya $(-66 \%$ at $20 \% ;-33.7 \%$ at $10 \%)$, Euphorbia milii (-37\% at 20\%), Psychotria carthagenensis $(-25.5 \%$ at $2.5 \%)$, Clusia variegate $(-22 \%$ at $20 \%)$, and Zamioculcas zamiifolia (-21.5\% at 20\%). Stimulator extracts were Mentha villosa at 10\% (+148\%) and 2.5\% (+131.5\%), followed by Aloe vera $(+123 \%$ at $5 \%)$, Schinus molle $(+112.5 \%$ at $10 \%)$, Schefflera arboricola $(+93.5 \%$ at $5 \%), C$. variegate $(+89 \%$ at $5 \%)$, and $S$. molle ( $+88 \%$ at $5 \%)$. Some extracts kept population stable throughout the experiment, presenting lower control indexes. Besides an additive effect, there was an individual influence of concentration or time on control.
\end{abstract}

KEYWORDS: pesticide potentiality; nematicide compounds; phytopathology.

\begin{abstract}
RESUMO: O controle de fitonematoides é muito difícil e requer uma combinaçáo de técnicas para ter sucesso. O controle alternativo via extrato vegetal pode resultar na descoberta de substâncias nematicidas. Esta pesquisa objetivou avaliar o efeito de 33 plantas submetidas à extraçáo aquosa contra Panagrellus redivivus in vitro. As concentraçôes foram preparadas a 1,$25 ; 2,5 ; 5 ; 10$; e $20 \%$. O monitoramento ocorreu em 0, 6, 12, 24 e 30 horas após a preparação. Para a contagem, foram considerados nematoides mortos subtraídos dos vivos. Nematoides jovens também foram contados, e a eficiência dos extratos foi expressa em porcentagem de controle ou de estímulo. Os dados foram submetidos à análise de variância. Resultados significativos foram analisados pelos testes de Scott-Knott (5\%) e análise de regressão múltipla. Foram observados extratos agindo como controladores, bem como estimuladores da reprodução de nematoides. A melhor performance de controle foi obtida por Carica papaya (-66\% a 20\%; $-33,7 \%$ a $10 \%)$, Euphorbia milii (-37\% a $20 \%)$, Psychotria carthagenensis $(-25,5 \%$ a $2,5 \%)$, Clusia variegate (-22 a 20\%) e Zamioculcas zamiifolia (-21,5\% a 20\%). Os extratos estimuladores foram Mentha villosa a 10\% (+148\%) e $2,5 \%(+131,5 \%)$, seguido por Aloe vera $(+123 \%$ a $5 \%)$, Schinus molle $(+112.5 \%$ a $10 \%)$, Schefflera arboricola $(+93.5 \%$ a $5 \%)$, C. variegate ( $+89 \%$ a $5 \%)$ e $S$. molle ( $+88 \%$ a $5 \%)$. Alguns extratos mantiveram a população estável durante todo o experimento, apresentando menores índices de controle. Além do efeito aditivo houve uma influência individual da concentração e do tempo no controle.
\end{abstract}

PALAVRAS-CHAVE: potencial pesticida; componentes nematicidas; fitopatologia.

\footnotetext{
'Universidade Estadual do Oeste do Paraná - Marechal Cândido Rondon (PR), Brazil ¿Universidade Federal do Paraná - Palotina (PR), Brazil

${ }^{3}$ Escola Superior de Agricultura "Luiz de Queiroz", Universidade de São Paulo - Pracicaba (SP), Brazil

${ }^{4}$ Centro Universitário Integrado - Londrina (PR), Brazil

${ }^{5}$ Universidade Estadual de Londrina - Londrina (PR), Brazil

*Corresponding author: cleo.lubian@gmail.com

Received on: 05/25/2018. Accepted on: 07/29/2019
} 


\section{INTRODUCTION}

Plants are affected by biotic and abiotic factors, which leads to reduced productivity levels. Among biotic factors, diseases caused by phytoparasitic nematodes compromise root functions in soil, as water and nutrients uptake and plant support (CAMPOS et al., 2011).

Meloidogyne is the most important genus followed by Heterodera, Globodera, and Pratylenchus (JONES et al., 2013). Nematoda phylum contains over 27,000 species already described (QUIST et al., 2015), including free-living nematodes and those that affect animals. Related to phytonematodes, over 4,100 species were registered, causing damage in crops, corresponding to roughly 80 billion dollars per year (DE ALMEIDA ENGLER; FAVERY, 2011).

There are few viable techniques to control nematodes in croplands, resorting on chemical method, although it presents a high toxicity level (NTALLI; CABONI, 2017). In this context, alternative methods have been deeply studied as alternative methods, especially considering environmental concerns and human health, focused on decreasing nematicides use (DIAS et al., 2016).

Plant extract is practiced since 1972 (NAKASHIMA; SHIMIZU, 1972), and stands out because of its potential molecules with nematicidal activity, derived from secondary metabolism. Both aqueous and alcoholic plant extract were studied against nematodes (TARIQ et al., 2009). Many oils not only repeal plagues, but also present contact and fumigant action on nematodes (ISMAN, 2000). Nematicide oils are especially important in small areas (GARDIANO et al., 2009).

Given the easy handling of Panagrellus redivivus in laboratory, research aimed at testing nematicidal activity of aqueous extract from 33 plant species against $P$. redivivus. We considered cultural knowledge to hypothesize that new plants may contain nematicidal substances.

\section{MATERIAL AND METHODS}

Samples of 33 plant species were collected during spring season. Some plants have their athelmintic activity already described in literature (Table 1).

Plants were sent to the Phytopathology Laboratory of Universidade Federal do Paraná - Setor Palotina. Leaves were cut off for extract preparing. In the case of Carica papaya, we used seeds. Plant parts were weighted $(40 \mathrm{~g})$ and mixed in $60 \mathrm{~mL}$ of distilled water in a blender for three minutes, then filtrated on gauze. The resulting liquid was stored into test tubes and frozen at $-20^{\circ} \mathrm{C}$ for two weeks. For evaluation, such tubes were defrosted naturally for 24 hours. Next, the liquid was centrifuged for five minutes at 4,000 rpm applied for decanting substances elimination.
Dilutions were prepared just before adding nematodes, obeying a serial dilution composed by $1 \mathrm{~mL}$ directly pipetted from each extract placed into small Petri plates $(60 \times 15 \mathrm{~mm})$, jointly with $1 \mathrm{~mL}$ of distilled water, representing the first dilution of $20 \%$ of extract concentration. The following dilutions were performed in the same pattern, at 10, 5, 2.5, and 1.25\% $(\mathrm{w} / \mathrm{v})$, with four repetitions each. Control treatment was composed of distilled water. The experimental design was in a completely randomized set in room temperature $\left(25 \pm 2^{\circ} \mathrm{C}\right)$.

Panagrellus redivivus were maintained in a creamy mixture of oat flour and distilled water. Their collection comprised nematodes climbing the boundaries of the container they were into. In sequence, nematodes were washed to be free from flour particles. Population was calibrated with water into a Bequer to enable the medium collection of 10 individuals in $20 \mu \mathrm{L}$ of water drew up by electronic micropipette. This water volume was completed with $80 \mu \mathrm{L}$ of distilled water, the final volume.

For nematode death (non-motile) evaluation, the initial number of nematodes added into Petri plates was identified on the top of each lid to allow a precise following counting in a stereo microscope. In addition, newborn nematodes (juveniles) were also counted to determine whether extracts would stimulate P. redivinus reproduction.

Data were registered in spreadsheets for posterior percentage calculation to set how effective or which stimulator each extract it was. Evaluation criterion considered the balance of alive nematodes at all evaluation times, of which values were subtracted from the initial ones.

The first evaluation occurred after adding nematodes to check any possible immediate effect of extracts on their behavior, considering the reaction pattern, especially as to how fast they moved. Next counting was conducted after 6, 12, 24, and 30 hours of interaction with each extract.

Data were submitted to variance analysis (ANOVA). In cases of significant results, Scott-Knott media test was applied at 5\% of error probability $(\mathrm{p}=0.05)$ to group extracts, according to their similarity. Extracts presenting distinguished effects were analyzed with the linear regression test $(\mathrm{p}=0.05)$, which was applied individually to time and dose factors to determine their particular influence on nematodes. As the dependent variable (alive nematodes) was explained by dose and time combined, the multiple regression test $(\mathrm{p}=0.05)$ was applied to all extracts to describe, mathematically, the relation between independent and dependent variables. The usefulness of regression prediction with the equation was set by determination coefficient. Tests were performed with SISVAR $5.6^{\oplus}$ program (FERREIRA, 2011).

\section{RESULTS}

ANOVA presented significance for time, dose, extract, extract* dose and extract ${ }^{*}$ time (data not presented). To these parameters, Scott-Knott media test was applied at $5 \%$ of probability. 
Concerning data presentation, negative signal before percentage values determines nematodes control, whereas the absence of signal represents stimulation on nematode reproduction.

At 0 hour, a maximum stimulation and reduction of 10 and $-14 \%$, respectively, was found (Table 2). However, the number expressed at this time refers to the real number of nematodes added to each extract; therefore, it is possible for eight nematodes to have been counted for Agave angustifolia, for example. Regardless of that, there was no significant difference at 0 hour, revealing no readily effects of any extract. Fluctuations as from six hours reveals the real effects of extracts. The highest control percentage was $-17.08 \%$, reached for Peschiera fuchsiaefolia at 30 hours; the highest stimulation, $170.42 \%$, was caused by Mentha villosa at 30 hours.

Extracts varied on effectiveness and many of them kept the nematode population stable throughout the experiment, such as Garcinia gardneriana, Psychotria carthagenensis, Bauhinia forficata, P. fuchsiaefolia, Genipa americana, Ligustrum lucidum,

Table 1. Common names, scientific names and botanical family of collected plants.

\begin{tabular}{|c|c|c|}
\hline Common name in Portuguese & Family & Scientific name \\
\hline Agave (Or) & Asparagaceae & Agave angustifolia Haw. (1812) \\
\hline Babosa (M) & Asphodelaceae & Aloe vera (L.) Burm. f. (1768) \\
\hline Bacupari (A) & Clusiaceae & Garcinia gardneriana (Planch. \&Triana) Zappi (1993) \\
\hline Cana-do-brejo (M) & Costaceae & Costus spicatus (Jacq.) Sw. (1788) \\
\hline Carqueja (M) & Asteraceae & Baccharis trimera (Less.) DC. (1836) \\
\hline Cedro (A) & Meliaceae & Cedrela fissilis Vell. (1825 [1829]) \\
\hline Cheflera (Or) & Araliaceae & Schefflera arboricola Hayata (1916) \\
\hline Aroeira-salsa (A) & Anacardiaceae & Schinus molle L. \\
\hline Clusia (Or) & Clusiaceae & Clusia variegate \\
\hline Comigo-ninguém-pode (Or) & Araceae & Dieffenbachia seguine (Jacq.) Schott (1829) \\
\hline Coroa-de-cristo (Or) & Euphobiaceae & Euphorbia milii Des Moul. (1826) \\
\hline Embaúba (A) & Urticaceae & Cecropia pachystachya Trécul. (1847) \\
\hline Erva-de-rato (W) & Rubiaceae & Psychotria carthagenensis Jacq. (1760) \\
\hline Espada-de-São-Jorge (Or) & Asparagaceae & Sansevieria trifasciata Prain (1903) \\
\hline Figo (S) & Moraceae & Ficus carica L. (1753) \\
\hline Forquilheira (M) & Apocynaceae & Peschiera fuchsiaefolia (A. DC.) Miers \\
\hline Guiné (M) & Petiveriaceae & Petiveria alliacea L. (1753) \\
\hline Hortelã (M) & Lamiaceae & Mentha villosa Huds. (1778) \\
\hline Jenipapo (A) & Rubiaceae & Genipa Americana L. (1759) \\
\hline Ligustre $(\mathrm{A})$ & Oleaceae & Ligustrum lucidum W. T. Aiton (1810) \\
\hline Losna (M) & Asteraceae & Artemisia absinthium (Mill.) DC. (1815) \\
\hline Mamão (A) & Caricaceae & Carica papaya L. (1753) \\
\hline Mandioca-brava (S) & Euphobiaceae & Manihot esculenta Crantz (1766) \\
\hline Maria-preta $(\mathrm{W})$ & Solanaceae & Solanum americanum Mill. (1768) \\
\hline Mulungu (Or/M) & Fabaceae & Erythrina verna Vell. (1825) \\
\hline $\operatorname{Noni}(\mathrm{A} / \mathrm{M})$ & Rubiaceae & Morinda citrifolia L. (1753) \\
\hline Pau-d'alho (A) & Petiveriaceae & Gallesia integrifolia (Spreng.) Harms (1934) \\
\hline Pata-de-vaca (A) & Fabaceae & Bauhinia forficata L. (1753) \\
\hline Pinhão-roxo (A) & Euphobiaceae & Jatropha gossypifolia L. (1866) \\
\hline Poejo (M) & Lamiaceae & Mentha sect. pulegium Coss. \& Germ. (1845) \\
\hline Sabugueira (M) & Adoxaceae & Sambucus nigra L. (1753) \\
\hline Trombetarosa (Or) & Solanaceae & $\begin{array}{l}\text { Brugmansia suaveolens (Humb. \& Bonpl. Ex Willd.) Sweet } \\
\qquad(1818)\end{array}$ \\
\hline Zamioculca (Or) & Araceae & Zamioculcas zamiifolia (Lodd.) Engl. (1905) \\
\hline
\end{tabular}

Or: ornamental; M: medicinal; A: arboreal; S: shrub; W: weed. 
Table 2. Classificatory ranking of extract effect under time influence expressed in percentage.

\begin{tabular}{|c|c|c|c|c|c|c|c|c|c|c|c|}
\hline \multirow[b]{2}{*}{ Plant Specie } & \multicolumn{10}{|c|}{ TIME (hours) } & \multirow[b]{2}{*}{$\begin{array}{l}\text { General } \\
\text { Average }\end{array}$} \\
\hline & 0 & $\begin{array}{c}\text { C or S } \\
(\%)\end{array}$ & 6 & $\begin{array}{c}\text { C or S } \\
(\%)\end{array}$ & 12 & $\begin{array}{c}\text { C or S } \\
(\%)\end{array}$ & 24 & $\begin{array}{c}\text { C or S } \\
(\%)\end{array}$ & 30 & $\begin{array}{c}\text { C or S } \\
(\%)\end{array}$ & \\
\hline $\begin{array}{l}\text { Agave } \\
\text { angustifolia }\end{array}$ & $8.583 \mathrm{Aa}$ & -14.2 & $8.583 \mathrm{Aa}$ & -14.17 & $9.041 \mathrm{Aa}$ & -9.59 & 13.667 Bc & 36.67 & $14.500 \mathrm{Bc}$ & 45 & 10.875 \\
\hline Aloe vera & $9.833 \mathrm{Aa}$ & -1.67 & 10.667 Aa & 6.67 & 14.208 Bb & 42.08 & $23.708 \mathrm{Cf}$ & 137.08 & $22.792 \mathrm{Cf}$ & 127.92 & 16.242 \\
\hline $\begin{array}{l}\text { Garcinia } \\
\text { gardneriana }\end{array}$ & $8.917 \mathrm{Aa}$ & -10.8 & $8.916 \mathrm{Aa}$ & -10.84 & $10.083 \mathrm{Aa}$ & 0.83 & $10.458 \mathrm{Aa}$ & 4.58 & $11.167 \mathrm{Ab}$ & 11.67 & 9.908 \\
\hline Costus spicatus & $8.708 \mathrm{Aa}$ & -12.9 & $10.000 \mathrm{Aa}$ & 0 & $11.750 \mathrm{Ba}$ & 17.5 & $16.667 \mathrm{Cd}$ & 66.67 & 18.208 Cd & 82.08 & 13.067 \\
\hline $\begin{array}{l}\text { Baccharis } \\
\text { trimera }\end{array}$ & $10.000 \mathrm{Aa}$ & 0 & $10.083 \mathrm{Aa}$ & 0.83 & 10.167 Aa & 1.67 & $15.125 \mathrm{Bc}$ & 51.25 & $24.625 \mathrm{Cg}$ & 146.25 & 14.000 \\
\hline Cedrera fissilis & $9.500 \mathrm{Aa}$ & -5 & $9.500 \mathrm{Aa}$ & -5 & $9.625 \mathrm{Aa}$ & -3.75 & $9.708 \mathrm{Aa}$ & -2.92 & $9.542 \mathrm{Aa}$ & -4.58 & 9.575 \\
\hline $\begin{array}{l}\text { Schefflera } \\
\text { arboricola }\end{array}$ & $10.000 \mathrm{Aa}$ & 0 & $11.000 \mathrm{Aa}$ & 10 & $13.250 \mathrm{Bb}$ & 32.5 & $20.875 \mathrm{Ce}$ & 108.75 & $25.333 \mathrm{Dg}$ & 153.33 & 16.092 \\
\hline Schinus molle & 10.208 Aa & 2.08 & $14.667 \mathrm{Bc}$ & 46.67 & $17.000 \mathrm{Cc}$ & 70 & $21.000 \mathrm{De}$ & 110 & $\mathrm{Cd}$ & 85.83 & 16.292 \\
\hline $\begin{array}{l}\text { Clusia } \\
\text { variegata }\end{array}$ & $9.958 \mathrm{Aa}$ & -0.42 & $10.917 \mathrm{Aa}$ & 9.17 & $12.375 \mathrm{Ab}$ & 23.75 & $18.583 \mathrm{Bd}$ & 85.83 & $19.583 \mathrm{Bd}$ & 95.83 & 14.283 \\
\hline $\begin{array}{l}\text { Dieffenbachia } \\
\text { seguine }\end{array}$ & $10.208 \mathrm{Aa}$ & 2.08 & $10.208 \mathrm{Aa}$ & 2.08 & $10.333 \mathrm{Aa}$ & 3.33 & $13.917 \mathrm{Bc}$ & 39.17 & $15.792 \mathrm{Bc}$ & 57.92 & 12.092 \\
\hline Euphorbia milii & $9.292 \mathrm{Aa}$ & -7.08 & $9.292 \mathrm{Aa}$ & -7.08 & $9.458 \mathrm{Aa}$ & -5.42 & $2 \mathrm{Aa}$ & 2.92 & $5 \mathrm{Ab}$ & 1 & 9.942 \\
\hline $\begin{array}{l}\text { Cecropia } \\
\text { pachystachya }\end{array}$ & $8.708 \mathrm{Aa}$ & -12.9 & $8.708 \mathrm{Aa}$ & -12.92 & 10.125 Aa & 1.25 & $15.875 \mathrm{Bc}$ & 58.75 & 16.625 Bc & 66.25 & 12.008 \\
\hline $\begin{array}{l}\text { Psychotria } \\
\text { carthagenensis }\end{array}$ & $9.208 \mathrm{Aa}$ & -7.92 & $9.333 \mathrm{Aa}$ & -6.67 & $9.417 \mathrm{Aa}$ & -5.83 & $9.333 \mathrm{Aa}$ & -6.67 & $8.917 \mathrm{Aa}$ & -10.83 & 9.242 \\
\hline $\begin{array}{l}\text { Sansevieria } \\
\text { trifasciata }\end{array}$ & 10.375 Aa & 3.75 & $10.958 \mathrm{Aa}$ & 9.58 & $11.083 \mathrm{Aa}$ & 10.83 & $11.292 \mathrm{Ab}$ & 12.92 & $11.333 \mathrm{Ab}$ & 13.33 & 11.008 \\
\hline Ficus carica & 10.9 & 9.58 & 12.6 & 26.25 & $12.833 \mathrm{Ab}$ & 28.33 & 11.2 & 12.08 & $A b$ & 6.67 & 11.658 \\
\hline $\begin{array}{l}\text { Peschiera } \\
\text { fuchsiaefolia }\end{array}$ & $10.042 \mathrm{Aa}$ & 0.42 & $10.042 \mathrm{Aa}$ & 0.42 & $10.208 \mathrm{Aa}$ & 2.08 & $9.292 \mathrm{Aa}$ & -7.08 & $8.292 \mathrm{Aa}$ & -17.08 & 9.575 \\
\hline $\begin{array}{l}\text { Petiveria } \\
\text { alliaceae }\end{array}$ & $9.667 \mathrm{Aa}$ & -3.33 & $9.667 \mathrm{Aa}$ & -3.33 & $10.875 \mathrm{Aa}$ & 8.75 & 10.333 Aa & 3.33 & 10.000 Aa & 0 & 10.108 \\
\hline Mentha villosa & $9.667 \mathrm{Aa}$ & -3.33 & 10.75 & 7.5 & $17.250 \mathrm{Bc}$ & 72.5 & $25.542 \mathrm{Cf}$ & 155.42 & $2 \mathrm{Ch}$ & 170.42 & 18.050 \\
\hline $\begin{array}{l}\text { Genipa } \\
\text { americana }\end{array}$ & $9.417 \mathrm{Aa}$ & -5.83 & $9.542 \mathrm{Aa}$ & -4.58 & $9.792 \mathrm{Aa}$ & -2.08 & $10.042 \mathrm{Aa}$ & 0.42 & $9.958 \mathrm{Aa}$ & -0.42 & 9.750 \\
\hline $\begin{array}{l}\text { Ligustrum } \\
\text { lucidum }\end{array}$ & $9.833 \mathrm{Aa}$ & -1.67 & $9.792 \mathrm{Aa}$ & -2.08 & $9.958 \mathrm{Aa}$ & -0.42 & $9.708 \mathrm{Aa}$ & -2.92 & 10.125 Aa & 1.25 & 9.883 \\
\hline $\begin{array}{l}\text { Artemisia } \\
\text { absinthium }\end{array}$ & $9.375 \mathrm{Aa}$ & -6.25 & $9.375 \mathrm{Aa}$ & -6.25 & $9.625 \mathrm{Aa}$ & -3.75 & $9.708 \mathrm{Aa}$ & -2.92 & $9.792 \mathrm{Aa}$ & -2.08 & 9.575 \\
\hline Carica papaya & $10.416 \mathrm{Aa}$ & 4.16 & $9.250 \mathrm{Aa}$ & -7.5 & $10.708 \mathrm{Aa}$ & 7.08 & $9.625 \mathrm{Aa}$ & -3.75 & $8.500 \mathrm{Aa}$ & -15 & 9.700 \\
\hline $\begin{array}{l}\text { Manihot } \\
\text { esculenta }\end{array}$ & $10.375 \mathrm{Aa}$ & 3.75 & $12.000 \mathrm{Ab}$ & 20 & $12.625 \mathrm{Bb}$ & 26.25 & $13.458 \mathrm{Bc}$ & 34.58 & $14.208 \mathrm{Bc}$ & 42.08 & 12.533 \\
\hline $\begin{array}{l}\text { Solanum } \\
\text { americanum }\end{array}$ & $11.000 \mathrm{Aa}$ & 10 & $11.000 \mathrm{Aa}$ & 10 & $11.625 \mathrm{Aa}$ & 16.25 & $11.417 \mathrm{Ab}$ & 14.17 & $10.000 \mathrm{Aa}$ & 0 & 11.008 \\
\hline Erythrina verna & $9.333 \mathrm{Aa}$ & -6.67 & $9.333 \mathrm{Aa}$ & -6.67 & $10.583 \mathrm{Aa}$ & 5.83 & $14.000 \mathrm{Bc}$ & 40 & $21.125 \mathrm{Ce}$ & 111.25 & 12.875 \\
\hline $\begin{array}{l}\text { Morinda } \\
\text { citrifolia }\end{array}$ & 10.667 Aa & 6.67 & $12.500 \mathrm{Bb}$ & 25 & $12.708 \mathrm{Bb}$ & 27.08 & $11.708 \mathrm{Bb}$ & 17.08 & 10.167 Aa & 1.67 & 11.550 \\
\hline $\begin{array}{l}\text { Gallesia } \\
\text { integrifolia }\end{array}$ & $10.000 \mathrm{Aa}$ & 0 & $10.667 \mathrm{Aa}$ & 6.67 & $11.292 \mathrm{Aa}$ & 12.92 & $11.833 \mathrm{Ab}$ & 18.33 & $12.333 \mathrm{Ab}$ & 23.33 & 11.225 \\
\hline
\end{tabular}


Table 2. Continuation.

\begin{tabular}{|c|c|c|c|c|c|c|c|c|c|c|c|}
\hline \multirow[b]{2}{*}{ Plant Specie } & \multicolumn{10}{|c|}{ TIME (hours) } & \multirow{2}{*}{$\begin{array}{l}\text { General } \\
\text { Average }\end{array}$} \\
\hline & 0 & $\begin{array}{c}\text { C or S } \\
(\%)\end{array}$ & 6 & $\begin{array}{c}\text { C or S } \\
(\%)\end{array}$ & 12 & $\begin{array}{c}\text { C or S } \\
(\%)\end{array}$ & 24 & $\begin{array}{c}\text { C or S } \\
(\%)\end{array}$ & 30 & $\begin{array}{c}\text { C or S } \\
(\%)\end{array}$ & \\
\hline $\begin{array}{l}\text { Bauhinia } \\
\text { forficata }\end{array}$ & $10.208 \mathrm{Aa}$ & 2.08 & 10.167 Aa & 1.67 & $10.458 \mathrm{Aa}$ & 4.58 & $10.583 \mathrm{Aa}$ & 5.83 & $10.542 \mathrm{Aa}$ & 5.42 & 10.392 \\
\hline $\begin{array}{l}\text { Jatropha } \\
\text { gossypifolia }\end{array}$ & $9.750 \mathrm{Aa}$ & -2.5 & $9.750 \mathrm{Aa}$ & -2.5 & $9.875 \mathrm{Aa}$ & -1.25 & $9.333 \mathrm{Aa}$ & -6.67 & $9.167 \mathrm{Aa}$ & -8.33 & 9.575 \\
\hline $\begin{array}{l}\text { Mentha sect. } \\
\text { pulegium }\end{array}$ & $9.625 \mathrm{Aa}$ & -3.75 & $9.542 \mathrm{Aa}$ & -4.58 & 10.167 Aa & 1.67 & $14.333 \mathrm{Bc}$ & 43.33 & 16.000 Bc & 60 & 11.933 \\
\hline $\begin{array}{l}\text { Sambucus } \\
\text { nigra }\end{array}$ & $9.833 \mathrm{Aa}$ & -1.67 & $9.958 \mathrm{Aa}$ & -0.42 & $10.583 \mathrm{Aa}$ & 5.83 & $12.208 \mathrm{Bb}$ & 22.08 & $12.208 \mathrm{Bb}$ & 22.08 & 10.958 \\
\hline $\begin{array}{l}\text { Brugmansia } \\
\text { suaveolens }\end{array}$ & $9.125 \mathrm{Aa}$ & -8.75 & $9.125 \mathrm{Aa}$ & -8.75 & $9.792 \mathrm{Aa}$ & -2.08 & $10.958 \mathrm{Ab}$ & 9.58 & $15.417 \mathrm{Bc}$ & 54.17 & 10.883 \\
\hline $\begin{array}{l}\text { Zamioculcas } \\
\text { zamiifolia }\end{array}$ & $9.583 \mathrm{Aa}$ & -4.17 & $9.750 \mathrm{Aa}$ & -2.5 & $11.125 \mathrm{Ba}$ & 11.25 & $11.547 \mathrm{Bb}$ & 15.47 & $12.250 \mathrm{Bb}$ & 22.5 & 10.851 \\
\hline C.V. (\%) & 30.84 & & & & & & & & & & \\
\hline Maximum & & 10 & & 46.67 & & 72.5 & & 155.42 & & 170.42 & 18.0502 \\
\hline Minimum & & -14.2 & & -14.17 & & -9.59 & & -7.08 & & -17.08 & 9.2416 \\
\hline
\end{tabular}

C: controller extract; S: stimulator extract. Means followed by the same lowercase letter in column and by the same capital letter in line did not differ significantly from each other with the Scott-Knott test, at $5 \%$ error probability.

Solanum americanum, Morinda citrifolia, Gallesia integrifolia, Sambucus nigra, and Brugmansia suaveolens. All others presented some variation at some point.

Extract concentration had more influence (Table 3) on control percentages than time (Table 2). Most lethal yields were caused by $C$. papaya $(-66 \%$ at $20 \% ;-33.7 \%$ at $10 \%)$, Euphorbia milii (-37\% at $20 \%)$, P. carthagenensis $(-25.5 \%$ at $2.5 \%)$, Clusia variegate (-22\% at $20 \%)$, Zamioculcas zamiifolia $(-21.5 \%$ at $20 \%)$, P. fuchsiaefolia (-20.5\% at $10 \%)$, and Cedrera fissilis $(-19.5 \%$ at $20 \%)$. Other extracts displaying control were Ligustrum lucidum (-18.5\% at 1.25\%), Artemisia absinthium $(-18.5 \%$ at $2.5 \%)$, C. fissilis (-17.5\% at $1.25 \%)$, Garcinia gardneriana $(-17 \%$ at $1.25 \%)$, Jatropha gossypifolia $(-17 \%$ at $2.5 \% ;-16 \%$ at $20 \%)$, and Petiveria alliaceae (-14\% at $20 \%)$. Others presented lower control indexes.

On the other hand, several extracts stimulated nematode reproduction, an undesirable reaction. In fact, most results varied from a slight to a huge reproduction increase, resulting in many juveniles. There were 24 extracts surpassing $50 \%$ on stimuli. M. villosa only stimulated nematodes, being higher at concentrations of $10 \%(148 \%)$, and $2.5 \%$ (131.5\%), followed by Aloe vera (123\% at 5\%), Schinus molle (112.5\% at $10 \%)$, Schefflera arboricola (93.5\% at 5\%), C. variegate (89\% at $5 \%$ ), and $S$. molle ( $88 \%$ at $5 \%)$. Concentration influenced extract effect, especially for $C$. variegate, that also had controlled nematodes (-22\% at $20 \%)$.

Surprisingly, among the plants randomly chosen, some ornamentals, such as C. variegate, A. angustifolia, E. milii, $Z$. zamiifolia, Dieffenbachia seguine, and Sansevieria trifasciata killed nematodes in a specific concentration, especially at $20 \%$, although they were stimulated in others. E. milii kept population low and stable in concentrations lower than $20 \%$. In the ornamental group, only B. suaveolens and S. arboricola did not control nematodes at any concentration (Table 3). P. carthagenensis controlled nematodes in all concentrations, but higher concentrations did not determine higher control percentages, revealing no linear relation between dose and its respective controlling percentage (Table 4).

In respect to regression analysis, ANOVA showed significance for time, dose and their interaction (data not presented). As a biological characteristic, extracts did not express linear behavior, fitting better at second degree polynomial equation (Table 4). Graphs allow a better individual interpretation of time, and dose influences contrasting extracts. There was an additional effect for M. villosa (Fig. 1A), where longer exposure time increased reproduction, as well as dose, although dose was not significant (Table 4). Doses of $C$. papaya influenced nematodes more as to death, whereas time only kept population stable (Fig. 1B). For $Z$. zamiifolia, nematodes were not stimulated for time or dose (Fig. 2A); on the other hand, time caused nematode reproduction for $C$. pachystachya, whereas dose had no significant effects on it (Fig. 2B).

After evaluating the effect of six concentrations, the balance of alive nematodes was influenced by this combination five times more, and both Scott-Knott and regression tests masked some remarkable results, such as for $C$. papaya, that controlled $100 \%$ of nematodes after 24 hours at $10 \% \mathrm{w} / \mathrm{v}$, and after 12 hours at $20 \% \mathrm{w} / \mathrm{v}$. 
Table 3. Classificatory ranking of extract reaction under dose influence expressed in percentage of effect.

\begin{tabular}{|c|c|c|c|c|c|c|c|c|c|c|c|}
\hline \multirow[b]{2}{*}{ Plant Specie } & \multicolumn{10}{|c|}{ CONCENTRATION (\%) } & \multirow[b]{2}{*}{$\begin{array}{l}\text { General } \\
\text { Average }\end{array}$} \\
\hline & 1,25 & $\begin{array}{c}\text { C or S } \\
(\%)\end{array}$ & 2,5 & $\begin{array}{c}\text { C or S } \\
(\%)\end{array}$ & 5 & $\begin{array}{c}\text { C or S } \\
(\%)\end{array}$ & 10 & $\begin{array}{l}\text { C or S } \\
(\%)\end{array}$ & 20 & $\begin{array}{l}\text { C or S } \\
(\%)\end{array}$ & \\
\hline $\begin{array}{l}\text { Agave } \\
\text { angustifolia }\end{array}$ & $13.200 \mathrm{Bc}$ & 32 & $9.750 \mathrm{Aa}$ & $-2,5$ & $11.650 \mathrm{Bb}$ & 16,5 & $11.150 \mathrm{Bc}$ & 11,5 & $9.000 \mathrm{AC}$ & -10 & 11,15 \\
\hline Aloe vera & $14.650 \mathrm{AC}$ & 46,5 & 17.250 Bd & 72,5 & $22.300 \mathrm{Cf}$ & 123 & $14.400 \mathrm{AC}$ & 44 & 18.350 Bf & 83,5 & 17,25 \\
\hline $\begin{array}{l}\text { Garcinia } \\
\text { gardneriana }\end{array}$ & $8.300 \mathrm{Aa}$ & -17 & $9.850 \mathrm{Aa}$ & $-1,5$ & 10.100 Aa & 1 & $9.650 \mathrm{Ab}$ & $-3,5$ & $11.050 \mathrm{Ad}$ & 10,5 & 9,85 \\
\hline Costus spicatus & $12.750 \mathrm{Ab}$ & 27,5 & 16.450 Bd & 64,5 & $11.750 \mathrm{Ab}$ & 17,5 & $14.750 \mathrm{Bf}$ & 47,5 & $11.950 \mathrm{Ae}$ & 19,5 & 12,75 \\
\hline $\begin{array}{l}\text { Baccharis } \\
\text { trimera }\end{array}$ & $17.300 \mathrm{Bd}$ & 73 & 14.300 Ac & 43 & $14.650 \mathrm{Ac}$ & 46,5 & 14.150 Ac & 41,5 & 13.100 Ae & 31 & 14,30 \\
\hline Cedrera fissilis & $10.950 \mathrm{Bb}$ & 9,5 & $10.500 \mathrm{Bb}$ & 5 & $9.200 \mathrm{Aa}$ & -8 & $8.250 \mathrm{Aa}$ & $-17,5$ & $8.050 \mathrm{AC}$ & $-19,5$ & 9,20 \\
\hline $\begin{array}{l}\text { Schefflera } \\
\text { arboricola }\end{array}$ & 17.750 Bd & 77,5 & $17.500 \mathrm{Bd}$ & 75 & 19.350 Be & 93,5 & $17.150 \mathrm{Be}$ & 71,5 & 14.300 Ae & 43 & 17,50 \\
\hline Schinus molle & $17.000 \mathrm{Bd}$ & 70 & $14.750 \mathrm{Ac}$ & 47,5 & $18.800 \mathrm{Be}$ & 88 & $21.250 \mathrm{Cf}$ & 112,5 & 15.450 Af & 54,5 & 17,00 \\
\hline $\begin{array}{l}\text { Clusia } \\
\text { variegata }\end{array}$ & $15.850 \mathrm{Bd}$ & 58,5 & $15.900 \mathrm{Bd}$ & 59 & $18.900 \mathrm{Ce}$ & 89 & 16.750 Be & 67,5 & $7.800 \mathrm{AC}$ & -22 & 15,90 \\
\hline $\begin{array}{l}\text { Dieffenbachia } \\
\text { seguine }\end{array}$ & $10.750 \mathrm{Ab}$ & 7,5 & $13.700 \mathrm{Bc}$ & 37 & $13.400 \mathrm{Bc}$ & 34 & $9.300 \mathrm{Ab}$ & -7 & $14.900 \mathrm{Bf}$ & 49 & 13,40 \\
\hline Euphorbia milii & $10.400 \mathrm{Bb}$ & 4 & $9.250 \mathrm{Ba}$ & $-7,5$ & $12.600 \mathrm{Bb}$ & 26 & $10.600 \mathrm{Bb}$ & 6 & $6.300 \mathrm{Ab}$ & -37 & 10,40 \\
\hline $\begin{array}{l}\text { Cecropia } \\
\text { pachystachya }\end{array}$ & $11.750 \mathrm{Ba}$ & 17,5 & $13.850 \mathrm{Ac}$ & 38,5 & $11.400 \mathrm{Ab}$ & 14 & $12.550 \mathrm{AC}$ & 25,5 & $12.000 \mathrm{Ae}$ & 20 & 12,00 \\
\hline $\begin{array}{l}\text { Psychotria } \\
\text { carthagenensis }\end{array}$ & $8.900 \mathrm{Aa}$ & -11 & $7.450 \mathrm{Aa}$ & $-25,5$ & $9.850 \mathrm{Aa}$ & $-1,5$ & $9.800 \mathrm{Ab}$ & -2 & $8.950 \mathrm{AC}$ & $-10,5$ & 8,95 \\
\hline $\begin{array}{l}\text { Sansevieria } \\
\text { trifasciata }\end{array}$ & $9.150 \mathrm{Aa}$ & $-8,5$ & $12.050 \mathrm{Bb}$ & 20,5 & 10.200 Aa & 2 & $10.350 \mathrm{Ab}$ & 3,5 & $13.800 \mathrm{Be}$ & 38 & 10,35 \\
\hline Ficus carica & 14.050 Bc & 40,5 & $11.250 \mathrm{Ab}$ & 12,5 & $11.350 \mathrm{Ab}$ & 13,5 & $12.750 \mathrm{Bc}$ & 27,5 & 10.050 Ad & 0,5 & 11,35 \\
\hline $\begin{array}{l}\text { Peschiera } \\
\text { fuchsiaefolia }\end{array}$ & $8.850 \mathrm{Aa}$ & $-11,5$ & $10.250 \mathrm{Aa}$ & 2,5 & $10.500 \mathrm{Aa}$ & 5 & $7.950 \mathrm{Aa}$ & $-20,5$ & $9.400 \mathrm{AC}$ & -6 & 9,40 \\
\hline $\begin{array}{l}\text { Petiveria } \\
\text { alliaceae }\end{array}$ & $13.350 \mathrm{Bc}$ & 33,5 & $9.400 \mathrm{Aa}$ & -6 & $8.950 \mathrm{Aa}$ & $-10,5$ & $9.850 \mathrm{Ab}$ & $-1,5$ & $8.600 \mathrm{AC}$ & -14 & 9,40 \\
\hline Mentha villosa & 16.350 Ad & 63,5 & $23.150 \mathrm{Be}$ & 131,5 & 16.000 Ad & 60 & $24.800 \mathrm{Bg}$ & 148 & $17.500 \mathrm{Af}$ & 75 & 17,50 \\
\hline $\begin{array}{l}\text { Genipa } \\
\text { americana }\end{array}$ & $9.300 \mathrm{Aa}$ & -7 & $11.100 \mathrm{Ab}$ & 11 & $9.150 \mathrm{Aa}$ & $-8,5$ & $9.550 \mathrm{Ab}$ & $-4,5$ & $8.900 \mathrm{AC}$ & -11 & 9,30 \\
\hline $\begin{array}{l}\text { Ligustrum } \\
\text { lucidum }\end{array}$ & $8.150 \mathrm{Aa}$ & $-18,5$ & $9.800 \mathrm{Aa}$ & -2 & 10.100 Aa & 1 & $10.700 \mathrm{Ab}$ & 7 & $10.050 \mathrm{Ad}$ & 0,5 & 10,05 \\
\hline $\begin{array}{l}\text { Artemisia } \\
\text { absinthium }\end{array}$ & $8.950 \mathrm{Aa}$ & $-10,5$ & $8.150 \mathrm{Aa}$ & $-18,5$ & $11.400 \mathrm{Bb}$ & 14 & $9.800 \mathrm{Bb}$ & -2 & $8.650 \mathrm{AC}$ & $-13,5$ & 8,95 \\
\hline Carica papaya & 14.900 Dc & 49 & $11.300 \mathrm{Cb}$ & 13 & $11.850 \mathrm{Cb}$ & 18,5 & $6.250 \mathrm{Ba}$ & $-37,5$ & $3.400 \mathrm{Aa}$ & -66 & 11,30 \\
\hline $\begin{array}{l}\text { Manihot } \\
\text { esculenta }\end{array}$ & 12.100 Ab & 21 & $11.600 \mathrm{Ab}$ & 16 & $10.400 \mathrm{Aa}$ & 4 & 14.750 Bf & 47,5 & $15.850 \mathrm{Bf}$ & 58,5 & 12,10 \\
\hline $\begin{array}{l}\text { Solanum } \\
\text { americanum }\end{array}$ & $11.400 \mathrm{Ab}$ & 14 & $10.650 \mathrm{Ab}$ & 6,5 & $11.350 \mathrm{Ab}$ & 13,5 & $9.700 \mathrm{Ab}$ & -3 & $12.450 \mathrm{Ae}$ & 24,5 & 11,35 \\
\hline Erythrina verna & $14.700 \mathrm{BC}$ & 47 & $17.750 \mathrm{Cd}$ & 77,5 & $13.300 \mathrm{Bc}$ & 33 & $10.450 \mathrm{Ab}$ & 4,5 & 10.550 Ad & 5,5 & 13,30 \\
\hline $\begin{array}{l}\text { Morinda } \\
\text { citrifolia }\end{array}$ & $12.900 \mathrm{Ab}$ & 29 & $12.300 \mathrm{Ab}$ & 23 & $9.950 \mathrm{Aa}$ & $-0,5$ & $11.800 \mathrm{Ac}$ & 18 & $11.850 \mathrm{Ae}$ & 18,5 & 11,85 \\
\hline $\begin{array}{l}\text { Gallesia } \\
\text { integrifolia }\end{array}$ & $12.200 \mathrm{Ab}$ & 22 & $12.300 \mathrm{Ab}$ & 23 & $11.300 \mathrm{Ab}$ & 13 & $9.950 \mathrm{Ab}$ & $-0,5$ & $11.100 \mathrm{Ae}$ & 11 & 11,30 \\
\hline
\end{tabular}


Table 3. Continuation.

\begin{tabular}{|c|c|c|c|c|c|c|c|c|c|c|c|}
\hline \multirow[b]{2}{*}{ Plant Specie } & \multicolumn{10}{|c|}{ CONCENTRATION (\%) } & \multirow{2}{*}{$\begin{array}{l}\text { General } \\
\text { Average }\end{array}$} \\
\hline & 1,25 & $\begin{array}{c}\text { C or S } \\
(\%)\end{array}$ & 2,5 & $\begin{array}{c}\text { C or S } \\
(\%)\end{array}$ & 5 & $\begin{array}{c}\text { C or S } \\
(\%)\end{array}$ & 10 & $\begin{array}{c}\text { C or S } \\
(\%)\end{array}$ & 20 & $\begin{array}{c}\text { C or S } \\
(\%)\end{array}$ & \\
\hline $\begin{array}{l}\text { Bauhinia } \\
\text { forficata }\end{array}$ & $10.600 \mathrm{Ab}$ & 6 & $10.950 \mathrm{AC}$ & 9,5 & $8.750 \mathrm{Aa}$ & $-12,5$ & $10.600 \mathrm{Ab}$ & 6 & $10.950 \mathrm{Ad}$ & 9,5 & 10,60 \\
\hline $\begin{array}{l}\text { Jatropha } \\
\text { gossypifolia }\end{array}$ & $11.800 \mathrm{Bb}$ & 18 & $8.300 \mathrm{Aa}$ & -17 & $9.000 \mathrm{Aa}$ & -10 & $9.450 \mathrm{Ab}$ & $-5,5$ & $8.400 \mathrm{Ac}$ & -16 & 9,00 \\
\hline $\begin{array}{l}\text { Mentha sect. } \\
\text { pulegium }\end{array}$ & $11.100 \mathrm{Ab}$ & 11 & $9.950 \mathrm{Aa}$ & $-0,5$ & $11.750 \mathrm{Ab}$ & 17,5 & $13.450 \mathrm{Bc}$ & 34,5 & $14.850 \mathrm{Bf}$ & 48,5 & 11,75 \\
\hline $\begin{array}{l}\text { Sambucus } \\
\text { nigra }\end{array}$ & $11.750 \mathrm{Ab}$ & 17,5 & $11.350 \mathrm{Ab}$ & 13,5 & $12.250 \mathrm{Ab}$ & 22,5 & $10.450 \mathrm{Ab}$ & 4,5 & $9.450 \mathrm{Ac}$ & $-5,5$ & 11,35 \\
\hline $\begin{array}{l}\text { Brugmansia } \\
\text { suaveolens }\end{array}$ & $11.950 \mathrm{Ab}$ & 19,5 & $10.500 \mathrm{Ab}$ & 5 & 10.700 Aa & 7 & $11.550 \mathrm{AC}$ & 15,5 & 10.100 Ad & 1 & 10,70 \\
\hline $\begin{array}{l}\text { Zamioculcas } \\
\text { zamiifolia }\end{array}$ & $12.200 \mathrm{Cb}$ & 22 & 10.150 Ba & 1,5 & 10.300 Ba & 3 & $14.100 \mathrm{Cd}$ & 41 & $7.850 \mathrm{Ac}$ & $-21,5$ & 10,30 \\
\hline C.V. (\%) & 30,84 & & & & & & & & & & \\
\hline Maximum & & 77,5 & & 131,5 & & 123 & & 148 & & 83,5 & 17,5 \\
\hline Minimum & & $-18,5$ & & $-25,5$ & & $-12,5$ & & $-37,5$ & & -66 & 8,95 \\
\hline
\end{tabular}

C: controller extract; S: stimulator extract. Means followed by the same lowercase letter in column and by the same capital letter in line did not differ significantly from each other with the Scott-Knott test, at $5 \%$ error probability.

All extracts showed significance to the combination between time and dose, although some of them presented no influence by dose or time variable by itself. For the extracts in which nematodes had no influence of the variables, population was kept stable throughout the experiment (Table 4).

\section{DISCUSSION}

The search for new plants with anthelmintic potential may be random or focused on no host species to phytonematodes, since these plants could contain nematicide compounds (MARTINS; SANTOS, 2016). Seen that, WIRATNO et al. (2009) tested nematicidal activity of 17 plant extracts against Meloidogyne incognita with an ethanolic extraction of chemical compounds, composing buds, leaves, flowers, roots, seeds, and stems. According to them, tobacco, clove and betelvine presented high toxicity levels, killing over $80 \%$ of nematodes at a $5 \mathrm{mg}$ $\mathrm{mL}^{-1}$ dosage, followed by sweet flag, pyrethrum, and citronella, whose control potential ranged from 10 to $20 \%$. In our study, no extract was more effective than $66 \%$. One explanation lies on the fact that extraction method changes effectiveness of secondary metabolites against phytopathogens, also related to the influence of temperature (VENTUROSO et al., 2010).

KLIMPEL et al. (2011) evaluated the effectiveness of 13 plant extracts submitted to different extraction procedures (aqueous, ethanolic, methanolic, or chloroform) against three nematodes species. According to them, each methodology eluted compounds in different grades, presenting a varied potential. Aqueous extracts performed better control in vitro, especially after 24 hours.

Given that, aqueous extract of Ficus carica allowed nematodes reproduction at any dosage and time (Tables 2 and 3), whereas its methanolic extraction and use against $P$. redivivus showed inconsistent findings, ranging from less than $1 \%$ (CUNHA et al., 2003) to $96.2 \%(5 \mathrm{mg} / \mathrm{mL}$ ) after 72 hours (LIU et al., 2011).

HONG et al. (2007) tested 30 plants with ethanolic extraction to set their control potential against Bursaphelenchus xylophilus and P. redivivus. Among them, Sapiumse biserum, Nerium indicum, Magnolia grandiflora, Michelia hedyosperma, Zingiber striolatum, Punica granatum, and Edgeworthia chrysantha killed over $40 \%$ nematodes within 24 hours. In this study, only $C$. papaya overcame this mortality percentage, whose anthelmintic effect is well known in literature, although its methanolic extract killed only $15.36 \%$ of the initial population of $P$. redivivus (20-30 individuals) after 48 hours (CUNHA et al., 2003).

Extraction methods seem to vary among plant species, once methanolic extracts prepared from 24 plants were used to verify their nematicide potential against $P$. redivivus, revealing a reduction of over $94 \%$ by Leucaena leucocephala and Paspalum notatum (CUNHA et al., 2003), whereas other six plant extracts varied their efficiency from 11.054 to $36.33 \%$ after 48 hours. These control indexes surpass most of ours, possibly because authors applied the Tukey test, that compares all possible treatment medias among themselves, two-by-two, and the Scott-Knott test groups treatments minimize variation within groups and maximize variation among groups to 
compare them without ambiguity, leading to a lesser groups number formation.

It shall be said that statistical analysis also plays an important role. The Scott-Knott test is ideal to compare a large number of treatments for grouping related media without ambiguity (BHERING et al., 2008), considering that other tests may present data overlap (CANTERI et al., 2001). Nonetheless, results were hidden by multiple comparison of media (Tables 2 and 3), because statistical breakdown evaluates general dose at each time (vice-versa), and, evidently, any concentration in earlier evaluation periods had less effects. This is why, statistically, the highest control did not exceed 60\% when it actually did happen, especially for $C$. papaya extract, that killed $100 \%$ of nematodes at $20 \% \mathrm{w} / \mathrm{v}$ after 12,24 and 30 hours.

Mortality rates increased during exposure time (ELBADRI et al., 2008). However, only few extracts fit in such pattern, especially $C$. papaya, $P$. fuchsiaefolia, and $P$. carthagenensis (Table 2).

Table 4. Multiple linear regression test results.

\begin{tabular}{|c|c|c|}
\hline Specie & Equation & $\mathbf{R}^{2}$ \\
\hline Carica papaya & $y=13.543^{* *}-0.493 x^{* *}-0.046 x^{2 n s}$ & 42.31 \\
\hline Agave angustifolia & $y=8.323^{* *}-0.109 x^{n s}+0.226 x^{2 * *}$ & 59.80 \\
\hline Aloe vera & $y=7.602^{* *}+0.193 x^{n s}+0.513 x^{2 * *}$ & 58.69 \\
\hline Garcinia gardneriana & $y=8.387^{* *}+0.065 x^{* *}+0.077 x^{2 * *}$ & 57.45 \\
\hline Costus spicatus & $y=8.300 * *-0.0054 x^{n s}+0.333 x^{2 * *}$ & 65.53 \\
\hline Baccharis trimera & $y=7.767^{* *}-0.030 x^{n s}+0.446 x^{2 * *}$ & 44.91 \\
\hline Cedrera fissilis & $y=10.471^{* *}-0.147 x^{* *}+0.004 x^{2 n s}$ & 69.97 \\
\hline Schefflera arboricola & $y=8.555^{* *}-0.012 x^{n s}+0.529 x^{2 * *}$ & 65.20 \\
\hline Schinus molle & $y=11.127^{* *}+0.150 x^{n s}+0.291 x^{2 * *}$ & 35.80 \\
\hline Clusia variegate & $y=10.923^{* *}-0.269 x^{n s}+0.354 x^{2 * *}$ & 39.85 \\
\hline Dieffenbachia seguine & $y=8.396^{* *}+0.131 x^{*}+0.198 x^{2 * *}$ & 54.75 \\
\hline Euphorbia milii & $y=10.209^{* *}-0.191 x^{* *}+0.067 x^{2 n s}$ & 33.87 \\
\hline Cecropia pachystachya & $y=7.467^{* *}+0.023 x^{n s}+0.305 x^{2 * *}$ & 66.61 \\
\hline Psychotria carthagenensis & $y=9.404^{* *}-0.007 x^{n s}-0.008 x^{2 n s}$ & 0.81 \\
\hline Sansevieria trifasciata & $y=9.570^{* *}+0.161 x^{* *}+0.028 x^{2 n s}$ & 53.38 \\
\hline Ficus carica & $y=12.614^{* *}-0.074 x^{n s}-0.033 x^{2 n s}$ & 9.03 \\
\hline Peschiera fuchsiaefolia & $y=10.174^{* *}-0.051 x^{n s}-0.056 x^{2 *}$ & 18.85 \\
\hline Petiveria alliaceae & $y=10.730 * *-0.130 x^{*}+0.016 x^{2 n s}$ & 16.98 \\
\hline Mentha villosa & $y=7.537^{* *}+0.201 x^{n s}+0.640 x^{2 * *}$ & 59.11 \\
\hline Genipa americana & $y=9.898^{* *}-0.068 x^{* *}+0.020 x^{2 n s}$ & 35.48 \\
\hline Ligustrum lucidum & $y=9.571^{* *}+0.036 x^{n s}+0.005 x^{2 n s}$ & 7.12 \\
\hline Artemisia absinthium & $y=9.625^{* *}-0.040 x^{n s}+0.015 x^{2 n s}$ & 6.99 \\
\hline Manihot esculenta & $y=9.184^{* *}+0.265 x^{* *}+0.114 x^{2 * *}$ & 62.06 \\
\hline Solanum americanum & $y=10.923^{* *}+0.060 x^{n s}-0.021 x^{2 n s}$ & 12.14 \\
\hline Erythrina verna & $y=8.863^{* *}-0.192 x^{n s}+0.364 x^{2 * *}$ & 46.73 \\
\hline Morinda citrifolia & $y=11.878^{* *}+0.014 x^{n s}-0.029 x^{2 n s}$ & 3.32 \\
\hline Bauhinia forficata & $y=10.040^{* *}+0.023 x^{n s}+0.014 x^{2 n s}$ & 7.12 \\
\hline Gallesia integrifolia & $y=10.422^{* *}-0.040 x^{n s}+0.073 x^{2 * *}$ & 42.81 \\
\hline Jatropha gossypifolia & $y=10.527^{* *}-0.099 x^{*}-0.022 x^{2 n s}$ & 22.95 \\
\hline Mentha sect. pulegium & $y=7.061^{* *}+0.236 x^{* *}+0.233 x^{2 * *}$ & 76.87 \\
\hline Sambucus nigra & $y=10.246^{* *}-0.095 x^{* *}+0.092 x^{2 * *}$ & 64.30 \\
\hline Brugmansia suaveolens & $y=8.447^{* *}-0.034 x^{n s}+0.184 x^{2 * *}$ & 42.73 \\
\hline Zamioculcas zamiifolia & $y=10.257^{* *}-0.107 x^{*}+0.089 x^{2 * *}$ & 29.98 \\
\hline
\end{tabular}

$R^{2}$ : coefficient of determination; ** significant with $p \leq 0.01$; " significant with $p \leq 0.05$, ns: non-significant $(p>0.05)$; $y$ : alive nematodes estimative; $\mathrm{x}$ : dose independent variable; $\mathrm{x}^{2}$ : time independent variable. 
As to ornamental plants, CUNHA et al. (2003) did not notice significant effects on $P$. redivivus control after 48 hours by methanolic extracts of Asparagus densiflorus, Pelargonium hortorum, Zinnia elegans, Tagetes erecta, and Euphorbia pulcherrima, which belongs to the Euphorbiaceae family, just like E. milii - that kept population low and stable until 12 hours (Table 2). However, the authors reported control set by Dendranthema grandiflorum (36.33\%), and Bougainvillea glabra (23.39\%). In addition, KLIMPEL et al. (2011) did not observe any immediate effect (0 hours) of aqueous extracts on nematodes movement, exactly like what we found.

Most authors do not consider nematodes reproduction on research, solely counting the killed ones. Despite that, we considered this an important factor, because some plant extracts may stimulate nematodes, as noticed by many species in different concentrations and times (Tables 1 and 2). Some of these extracts had killed some individuals, but stimulated them more, and the final balance of alive nematodes masked those that were dead.

ELBADRI et al. (2008) tested methanol and hexane extracts $(0.05 \%$ concentration) of 21 medicinal plants for their toxicity against $M$. incognita. Nematodes were sensitive to all of them at some level, mainly after 72 hours. In this study, all concentrations of Baccharis trimera, M. villosa, and A. vera led to an increase of over than $30 \%$ in nematode reproduction (Table 3). Petiveria alliaceae had controlled over $10 \%$ of nematodes at 5 and $20 \% \mathrm{w} / \mathrm{v}$ concentrations, whereas A. absinthium did not control only at $5 \% \mathrm{w} / \mathrm{v}$, and $M$. sect. pulegium had irrelevant control $(0.5 \%)$ at $2.25 \% \mathrm{w} / \mathrm{v}$.

MARTINS; SANTOS (2016) prepared aqueous extracts of 10 medicinal plants to confront with $M$. incognita race 2 for 48 hours, counting inactive nematodes. Juveniles were put into water for 24 hours to analyze any eventual recovery. According to them, Eclipta alba, Ocimum basilicum, Artemisia vulgaris, Justicia pectoralis var. stenophylla, Spigelia
A



B

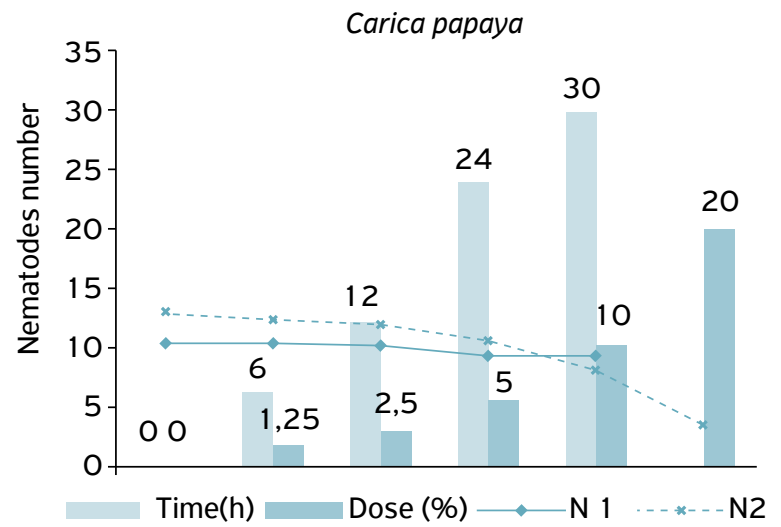

N1: alive nematodes influenced by time; N2: alive nematodes influenced by dose.

Figure 1. Mentha villosa (A) and Carica papaya (B) extracts, respectively.

A

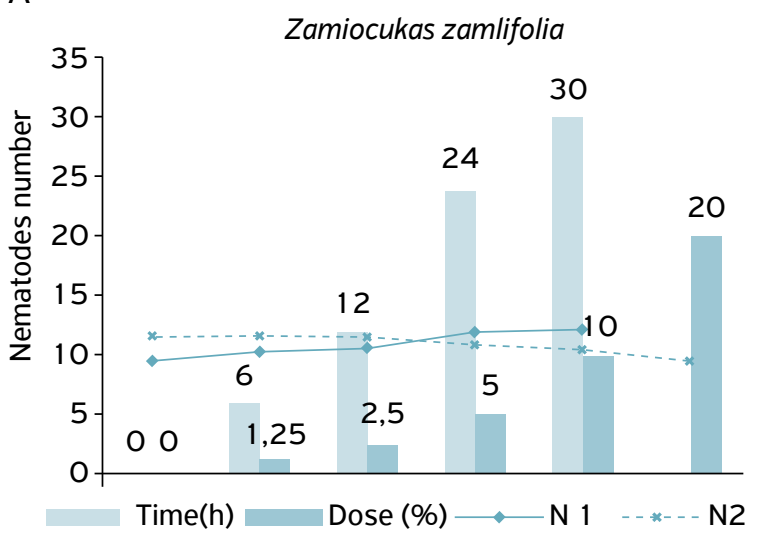

B

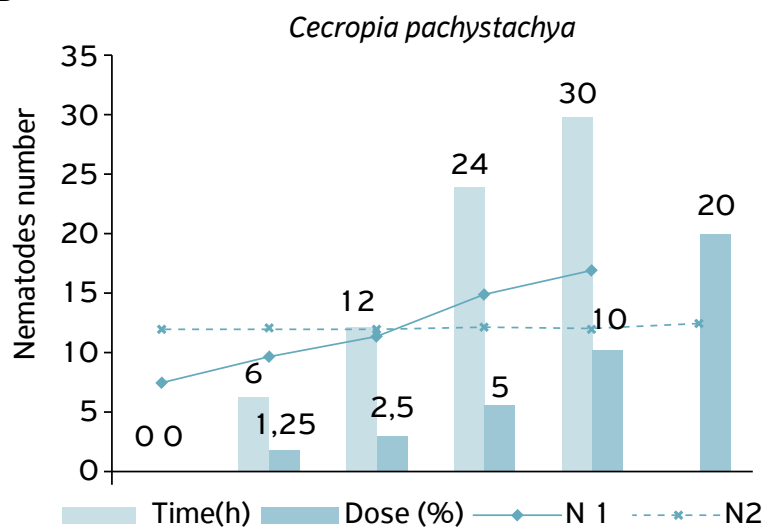

N1: alive nematodes influenced by time; N2: alive nematodes influenced by dose.

Figure 2. Zamioculcas zamiifolia (A) and Cecropia pachystachya (B) extracts, respectively. 
anthelmia, and Chenopodium ambrosioides killed over $75 \%$ of nematodes at the concentration of $10 \%$, prepared by either infusion or maceration. Nonetheless, M. x villosa was the less effective extract against $M$. incognita race 2 , and a stimulator to $P$. redivivus at any concentration (Table 3 ).

Panagrellus redivivus as a free-living nematode moves freely in water; the same behavior was seen when nematodes were exposed to many extracts, especially the stimulator ones. This way, it was easy to check dead individuals, because their body got very strict or even end-curled, without any physical moves. WIRATNO et al. (2009) reported shape changings, depending on the plant extract. An excessive movement for stimulator extracts was observed, to which the presence of many juveniles was very common, but it did not provoke death, unlikely noticed by WIRATNO et al. (2009). MARTINS; SANTOS (2016) demonstrated differences on nematicide and static effects.

ELBADRI et al. (2008) assumed that all extracts from seed had a higher nematicidal activity, matching with those we found (C. papaya). Seeds present higher chemical concentration of many soluble substances, such as alkaloids, tannin, phenolic compounds, and others (MARCOS FILHO, 2005).

In vitro evaluations aim at ranking extract plants to screen the best ones for further tests. MATEUS et al. (2014) evaluated the effect of biweekly application of Erythrina mulungu Mart. ex Benth aqueous extract, observing a reduction of $40 \%$ in $M$. incognita infectiveness in tomato roots, and $97.1 \%$ less eggs. This activity against nematodes is believed to result from secondary metabolites. Our in vitro results revealed the time factor, increasing $P$. redivivus population when treated with Erythrina verna (Table 4).
FERREIRA et al. (2013) tried aqueous extracts of Sphagneticola trilobata, Tagetes patula, Tithonia diversifolia, Tridax procumbens, Unxia suffruticosa, and Zinnia peruviana on $M$. incognita, recording eggs outbreak over $85 \%$ in vitro; when they were tested in tomato plants, no statistical difference for root weight and nematode reproduction index was noticed, compared to treatment with water (control). Therefore, our results are promising, although they may not display the same reaction in in vivo situations (KLIMPEL et al., 2011), demanding future assays.

In summary, $P$. redivivus was better controlled by Carica papaya, Euphorbia milii, Psychotria carthagenensis, Clusia variegate, Zamioculcas zamiifolia, Peschiera fuchsiaefolia, and Cedrera fissilis aqueous extracts, and highly stimulated by Mentha villosa, Aloe vera, Schinus molle, and Schefflera arboricola.

The hypothesis was confirmed. because some ornamental plants randomly chosen showed a control effect on P. redivivus, indicating the existence of new nematicides compounds. We first reported the action of $C$. variegate, $Z$. zamiifolia, and $P$. carthagenensis on $P$. redivivus.

For some extracts, dose and time had a concomitant influence on nematodes control, whereas for others, only one of such parameters was significant.

\section{ACKNOWLEDGEMENTS}

Universidade Federal do Paraná - Setor Palotina supported research.

\section{REFERENCES}

BHERING, L.L.; CRUZ, C.D.; VASCONCELOS, E.S.; FERREIRA, A.; RESENDE JUNIOR, M.F.R. Alternative methodology for Scott-Knott test. Crop Breeding and Applied Biotechnology, v.8, n. 1, p.9-16, 2008. http://dx.doi.org/10.12702/1984-7033.v08n01 aO2

CAMPOS, H.D.; CAMPOS, V.P.; SILVA, J.R.C.; SILVA, L.H.C.P.; COSTA, L.S.A.S.C.; TERRA, W.C. Atração e penetração de Meloidogyne javanica e Heterodera glycines em raízes excisadas de soja. Ciência Rural, v.41, n.9, p.1496-1502, 2011 . http://dx.doi. org/10.1590/SO103-84782011000900002

CANTERI, M.G.; ALTHAUS, R.A.; VIRGENS FILHO, J.S.; GIGLIOTI, E.A.; GODÓI, C.V. Sasm-Agri-Sistema para análise e separação de médias em experimentos agrícolas pelos métodos de ScottKnott, Tukey e Duncan. Revista Brasileira de Agrocomputação, v.1, n.2, p.18-24, 2001.
CUNHA, F.R.; OLIVEIRA, D.F.; CAMPOS, V.P. Extratos vegetais com propriedades nematicidas e purificação do princípio ativo do extrato de Leucaena leucocephala. Fitopatologia Brasileira, v.28, n.4, p.438-441, 2003. http://dx.doi.org/10.1590/ S0 $100-41582003000400017$

DE ALMEIDA ENGLER J., FAVERY, B. The plant cytoskeleton remodelling in nematode induced feeding sites. In: JONES, J.; GHEYSEN, G.; FENOLL, C. Genomics and Molecular Genetics of Plant-Nematode Interactions. Dordrecht: Springer, 2011 . p.369393. https://doi.org/10.1007/978-94-007-0434-3_18

DIAS, M.H.; BARBOSA, J.A.; PETERS, F.F.; STANGARLIN, J.R.; ESTEVES, R.L. Controle alternativo de Meloidogyne incognita em tomateiro. Scientia Agraria Paranaensis, v.15, n.4 p.421-426, 2016. http://dx.doi.org/10.1818/sap.v15i4.12491 
ELBADRI, G.A.; LEE, D.W.; PARK, J.C.; YU, H.B.; CHOO, H.Y. Evaluation of various plant extracts for their nematicidal efficacies against juveniles of Meloidogyne incognita. Journal of AsiaPacific Entomology, v.11, n.2, p.99-102, 2008. https://doi. org/10.1016/j.aspen.2008.04.004

FERREIRA, D. F. Sisvar: um sistema computacional de análise estatística. Ciência eAgrotecnologia, v.35, n.6, p.1039-1042. 2011. http://dx.doi.org/10.1590/S1413-70542011000600001

FERREIRA, I.C.M.; SILVA, G.S.; NASCIMENTO, F.S. Efeito de extratos aquosos de espécies de Asteraceae sobre Meloidogyne incognita. Summa Phytopathologica, v.39, n.1, p.40-44. 2013. http://dx.doi.org/10.1590/SO100-54052013000100007

GARDIANO, C.G.; FERRAZ, S.; LOPES, E.A.; FERREIRA, P.A.; AMORA D.X.; FREITAS, L.G. Evaluation of plant aqueous extracts, added into the soil on Meloidogyne javanica (Treub, 1885) Chitwood, 1949. Ciências Agrárias, v.30, n.3, p.551-556, 2009. http:// dx.doi.org/10.5433/1679-0359.2009v30n3p551

HONG, L.; LI, G.; ZHOU, W.; WANG, X.; ZHANG, K. Screening and isolation of a nematicidal sesquiterpene from Magnolia grandiflora L. Pest Management Science, v.63, n.3, p.301-305, 2007. https:// doi.org/10.1002/ps.1337

ISMAN, M.B. Plant essential oils for pest and disease management. Crop Protection, v.19, n.8-10, p.603-608, 2000. https://doi. org/10.1016/SO261-2194(O0)00079-X

JONES, J.T.; HAEGEMAN, A.; DANCHIN, E.G.J.; GAUR, H.S.; HELDER, J.; JONES, M.G.K.; KIKUCHI, T.; MANZANILLA-LÓPEZ, R.; PALOMARES-RIUS, J.E.; WESEMAEL, W.I.M.M.L.; PERRY, R.N. Top 10 plant-parasitic nematodes in molecular plant pathology. Molecular Plant Pathology, v.14, n.9, p.946-961, 2013. https:// doi.org/10.1111/mpp.12057

KLIMPEL, S.; ABDEL-GHAFFAR, F.; AL-RASHEID. K. A. S.; AKSU, G., FISCHER, K.; STRASSEN, B.; MEHLHORN, H. The effects of different plant extracts on nematodes. Parasitology Research, v. 108, n.4, p.1047-1054, 2011 . https://doi.org/10.1007/ s00436-010-2168-4

LIU, F.; YANG, Z.; ZHENG, X.; LUO, S.; ZHANG, K.; LI, G. Nematicidal coumarin from Ficuscarica L. Journal of Asia-Pacific Entomology, v. 14, n.1, p.79-81, 2011 . https://doi.org/10.1016/j. aspen.2010.10.006
MARCOS FILHO, J. Composição química de sementes (reservas armazenadas). In: MARCOS FILHO, J. Fisiologia de sementes de plantas cultivadas. Piracicaba: Fealq, 2005. 495p.

MARTINS, M.C.B.; SANTOS, C.D.G. Ação de extratos de plantas medicinais sobre juvenis de Meloidogyne incognita raça 2. Revista Ciência Agronômica, v.47, n.1, p.135-142, 2016. https://doi. org/10.5935/1806-6690.20160016

MATEUS, M.A.F.; FARIA, C.M.D.R.; BOTELHO, R.V.; DALLEMOLEGIARETTA, R.; FERREIRA, S.G.M.; ZALUSKI, W.L. Extratos aquosos de plantas medicinais no controle de Meloidogyne incognita (Kofoid e White, 1919) Chitwood, 1949. Bioscience Journal, v.30, n.3, p.730-736, 2014.

NAKASHIMA, Y.; SHIMIZU, K. Antitelmintic activity of Thujopsis dolabrata var. Hondai. III. Components with a termiticidal activity. Miyazaki Daigaku Nogakubu Kenkyu Hokoku, Bulletin of the Faculty of Agriculture, v.19, p.251-259, 1972.

NTALLI, N.G.; CABONI, P. A review of isothiocyanates biofumigation activity on plant parasitic nematodes. Phytochemistry Reviews, v. 16, n.5, p.827-834, 2017.https://doi.org/10.1007/s1 1 101-017-9491-7

QUIST, C.W.; SMANT, G.; HELDER, J. Evolution of plant parasitism in the phylum Nematoda. Annual Review of Phytopathology, v.53, n.1, p.289-310, 2015. https://doi.org/10.1146/ annurev-phyto-080614-120057

TARIQ, K.A.; CHISHTI, M.Z.; AHMAD, F.; SHAWL, A.S. Anthelmintic activity of extracts of Artemisia absinthium against ovine nematodes. Veterinary Parasitology, v. 160, n.1-2, p.83-88, 2009. https://doi.org/10.1016/j.vetpar.2008.10.084

VENTUROSO, L.R.; BACCHI, L.M.A.; GAVASSONI, W.L.; PONTIM, B.C.A.; CONUS, L.A. Influência de diferentes metodologias de esterilização sobre a atividade antifúngica de extratos aquosos de plantas medicinais. Revista Brasileira de Plantas Medicinais, v.12, n.4, p.499-505, 2010. http://dx.doi.org/10.1590/ S1516-05722010000400014

WIRATNO, W.; TANIWIRYONO, D.; VAN DEN BERG, H.; RIKSEN, J.A.G.; RIETJENS, I.M.C.M.; DJIWANTIA, S.R.; KAMMENGA, J.E.; MURK, A.J. Nematicidal activity of plant extracts against the root-knot nematode, Meloidogyne incognita. The Open Natural Products Journal, v.2, p.77-85, 2009. https://doi. org/10.2174/1874848100902010077 